\title{
Combined sterile insect technique and incompatible insect technique: sex separation and quality of sterile Aedes aegypti male mosquitoes released in a pilot population suppression trial in Thailand
}

Patttamaporn Kittayapong ${ }^{1,2^{*}}$, Nuanla-ong Kaeothaisong ${ }^{1}$, Suwannapa Ninphanomchai ${ }^{1}$ and Wanitch Limohpasmanee ${ }^{3}$

\begin{abstract}
Background: The sterile insect technique (SIT), which is based on irradiation-induced sterility, and incompatible insect technique (IIT), which is based on Wolbachia-induced cytoplasmic incompatibility (a kind of male sterility), have been used as alternative methods to reduce mosquito vector populations. Both methods require the release of males to reduce fertile females and suppress the number of natural populations. Different techniques of sex separation to obtain only males have been investigated previously. Our work involves an application of mechanical larval-pupal glass separators to separate Wolbachia-infected Aedes aegypti males from females at the pupal stage, prior to irradiation, and for use in a pilot field release and to assess the quality of males and females before and after sex separation and sterilization.

Results: This study was the first to demonstrate the efficiency of mechanical glass separators in separating males for use in an Ae. aegypti suppression trial by a combined SIT/IIT approach. Our results indicated that male and female pupae of Wolbachia-infected Ae. aegypti mosquitoes were significantly different $(p<0.05)$ in weight, size, and emergence-time, which made it easier for sex separation by this mechanical method. During the pilot field release, the percentage of female contamination was detected to be quite low and significantly different between the first $(0.10 \pm 0.13)$ and the second $(0.02 \pm 0.02)$ twelve-week period. Both males and females were almost completely sterile after exposure to $70 \mathrm{~Gy}$ irradiation dose. We observed that both irradiated Wolbachia-infected males and females survived and lived longer than two weeks, but males could live longer than females $(p<0.05)$ when they were irradiated at the same irradiation dose. When comparing irradiated mosquitoes with non-irradiated ones, there was no significant difference in longevity and survival-rate between those males, but non-irradiated females lived longer than irradiated ones $(p<0.05)$.

(Continued on next page)
\end{abstract}

\footnotetext{
* Correspondence: pkittayapong@gmail.com

${ }^{1}$ Center of Excellence for Vectors and Vector-Borne Diseases, Faculty of Science, Mahidol University at Salaya, Nakhon Pathom 73170, Thailand ${ }^{2}$ Department of Biology, Faculty of Science, Mahidol University, Bangkok 10400, Thailand

Full list of author information is available at the end of the article
} 
(Continued from previous page)

Conclusion: Mechanical sex separation by using a larval-pupal glass separator was practically applied to obtain only males for further sterilization and open field release in a pilot population suppression trial of Ae. aegypti in Thailand. Female contamination was detected to be quite low, and skilled personnel can reduce the risk for female release. The irradiated Wolbachia-infected females accidentally released were found to be completely sterile, with shorter life span than males.

Keywords: Mosquito vector, Pupal size, SIT, Sterility, Longevity, Wolbachia, IIT

\section{Introduction}

Dengue, chikungunya and Zika virus infections are mosquito-borne diseases that pose major public health problems in many countries where Aedes aegypti are dominant mosquito vectors. Since traditional vector control strategies do not provide satisfactory results, alternative eco-friendly techniques have been proposed to control mosquito vectors in many countries, including the sterile insect technique (SIT) as a component of an area-wide integrated vector management (AW-IVM) programme [1].

Another approach in sterilizing males is to exploit the phenomenon of Wolbachia-induced cytoplasmic incompatibility $(\mathrm{CI})$, which is expressed as embryonic lethality induced through mating between Wolbachia-infected males and uninfected females, or females infected with different Wolbachia strains $[1,2]$. Recently, a combined sterile insect technique and incompatible insect technique (SIT/ IIT) has been proposed as a means to introduce sterility into target populations of insect pests and disease vectors, including mosquitoes [3, 4]. In general, Wolbachia-infected females could be sterilized with a minimum dose of radiation that leads to complete sterility. As a result, any accidentally released females should be sterile and this would eliminate the risk of replacement of natural populations with Wolbachia-infected ones [1, 5-10].

Population suppression using the combined SIT/IIT approach requires release of a large number of male mosquitoes; therefore, an efficient separation between males and females is essential in order to produce and release only sterile males into the environment. Many studies have attempted to develop sex separation methods, based on biological, genetic and transgenic approaches, in order to support the application of SIT in mosquito control. Sieving technique was introduced by taking into account size difference between male and female pupae $[11,12]$. The development of genetic sexing strains (GSS) as well as other sex separation strategies are currently under development and/or refinement but none of them have so far succeeded to eliminate the females in order to achieve male only releases for SIT or other related applications [13-15].

Sterility in wild populations could be introduced on the condition that sterile male mosquitoes are of good quality and are able to compete with wild males. Previous studies have reported that the effect of irradiation and/or Wolbachia infection is minimal, if any, on the biological quality of Aedes albopictus by assessing traits such as egg-hatching rate, survival of pupae and adults, sex ratio, duration of larval stages, time to adult emergence, wing length, female fecundity, longevity, sterility and mating competitiveness $[8,16,17]$. In this study, and in the frame of a combined SIT/IIT population suppression trial in Thailand, we report the quality of Wolbachia-infected Ae. aegypti male and female pupae in term of differences in weight, size and developmental-time following sex separation. The quality of irradiated emerged males, i.e. sterility, survival and longevity that were important for the success of the suppression trial, was also investigated.

\section{Methods}

\section{Rearing of Aedes aegypti mosquito colony}

Aedes aegypti mosquitoes, used in the present study, were originally collected from villages in Pleang Yao District, Chachoengsao Province, while Aedes albopictus were collected from rubber plantations from the same area. A Wolbachia-transinfected Ae. aegypti colony was obtained from direct microinjection using the Aedes aegypti mosquito colony and Wolbachia strains from the Ae. albopictus collected from the same origin as those reported in Ruang-areerate and Kittayapong (2006) [18]. The establishment and characteristics of this Wolbachia-infected mosquito strain were demonstrated in Ruang-areerate and Kittayapong (2006) [18].

In these experiments, mosquitoes were reared in an aluminum cage sized $30 \mathrm{~cm}$ x $30 \mathrm{~cm}$ x $30 \mathrm{~cm}$, fed with $10 \%$ sucrose solution with $75 \pm 2 \%$ humidity, $27 \pm 2$ 。 $\mathrm{C}$, at a photoperiod of L12:D12 in a screen climatic control insectary at the Center of Excellence for Vectors and Vector-Borne Diseases, Faculty of Science, Mahidol University at Salaya, Nakhon Pathom, Thailand. Female mosquitoes were fed with pig blood, obtained from a qualified slaughterhouse, for 3-4 consecutive days after mating. The Hemotek blood-feeding system (Hemotek Ltd., UK), containing $20 \mathrm{ml}$ of pig blood, was placed on top of the cage for 1-2 hours of each feeding cycle. Egg papers were placed in the 
containers inside the cage following blood-feeding. After 3-4 days, the egg papers were then collected, dried and transferred into glass containers with screw-top covers filled with deionized water for egg hatching. After the eggs were hatched into the first-instar larvae, they were counted and transferred into plastic trays sized $32 \mathrm{~cm} \times$ $42 \mathrm{~cm} \times 5 \mathrm{~cm}$, each containing about 2000 larvae. After egg hatching, larval diet was provided daily, at a total quantity of $6.5 \mathrm{~g}$. The larval diet composed of mixed fish meal (Chanpongcharoen Kankaset Supplier, Thailand), pork liver powder and yeast (Saccharomyces cerevisiae) (Cheese Powder Supplier, Thailand) at a ratio of 5:4:1 respectively. No larval diet was given when larvae reached the pupal stage, which took about 6-7 days. Pupae were then placed in plastic containers prior to sex separation.

\section{Mechanical separation of male and female pupae}

Pupae were separated into different layers of males and females using larval-pupal glass separators (Model 5412, John W. Hock Company, Gainesville, FL, USA). Each unit consisted of horizontal aluminum plates supporting two glass panels that formed between them an adjustable, downward-pointing, wedge-shaped space into which the pupae could be filled. The numbers of pupae were thus separated on the basis of size by regulating the thickness and angle of the wedge-shaped space by means of four control knobs in each of the four angles. The lower opening was adjusted so that the larger female pupae were retained in a layer in the tapering space between the panels of glasses. The smaller male pupae were drained through into a receiving container placed below. The operation was completed by opening the wedge and flushing the female pupae into a second receiving container.

For each sex separation, one liter of water that contained about 1500 to 2000 mixed male and female pupae were introduced into the system. Adjustment of the glass panels was performed gradually, and water circulation was supplied all along the process in order to push and wash the pupae down into the container. The pupae maintained between the plates was varied by adjusting the angle of the plates. The smaller male pupae were flushed out first and then collected in plastic containers, whereas the bigger female pupae were collected after, and the cycle continued. One cycle of 1500-2000 pupae varied on average between 2-5 minutes, but it could take a longer time if there was a mixture of larvae inside. After counting, the male pupae were transferred into a plastic cup for further transportation to the radiation source. The process took place once a week from 09.00 - $11.00 \mathrm{am}$, in order to separate male and female pupae. In our study, 24 replicates were conducted, with the total numbers ranging from 9000 to $25,000(18,245 \pm 4,973)$ male pupae that were separated and transported for sterilization by irradiation and then the later emerged adult males released at the field site.

\section{Quality of male and female pupae: weight, size and emergence time}

After sex separation, Wolbachia-infected Ae. aegypti male and female pupae were introduced into plastic containers for further measurement and observation. They were then counted by using simple manual laboratory counting equipment. One thousand male or female pupae were separately placed in each plastic container half filled with water. A total number of 6 containers containing either male or female pupae were weighted and recorded in order to determine the weight of the pupae.

Three morphological characteristics, i.e. cephalothorax, abdomen and total length, were measured in 60 male and 60 female pupae $[19,20]$ in order to assess the difference in size. Each pupa was individually collected by using a dropper and was transferred into a small cup containing cold water $\left(4 \pm 2{ }^{\circ} \mathrm{C}\right)$ to make it immobilized. Then each pupa was placed on a glass slide and measured by the Olympus DP70 microscope (Tokyo, Olympus Corp.) using the DP Controller software (@2000 Olympus Optical Co., Ltd.).

In order to observe the developmental time, 1500 males and 1500 females of irradiated Wolbachia-infected pupae collected by using the dropper were transferred into a plastic bowl of $470 \mathrm{~cm}^{3}$ in volume and filled with $390-400 \mathrm{~cm}^{3}$ of water. The plastic bowl was then kept in a cage sized $30 \mathrm{~cm} \times 30 \mathrm{~cm} \times 30 \mathrm{~cm}$ and left in a screened insectary at a temperature of $27 \pm 2{ }^{\circ} \mathrm{C}, 75 \pm 2 \%$ humidity and a photoperiod of L12:D12. A ten percent sucrose solution was provided inside the cage for emerged adult mosquitoes. Emerged pupae were observed from the onset of adult emergence (day 0 ) for five consecutive days (days 1, 2, 3, 4, 5). Three replicates of 500 male and 500 female pupae each were observed in this experiment.

\section{Sterilization of male pupae and screening for female contamination}

Emerged Wolbachia-infected Aedes aegypti male pupae up to one day old were placed in plastic containers, each of $122.66 \mathrm{~cm}^{3}$ in volume (diameter $12.5 \mathrm{~cm}$, height $14.5 \mathrm{~cm}$ ) and with water of $62 \mathrm{~cm}^{3}$ in volume, prior to transportation to the radiation source. These plastic containers filled with male pupae were transported by air-conditioned car from the laboratory at Mahidol University Salaya Campus, Nakhon Pathom Province to the Thailand Institute of Nuclear Technology (Public Organization) (TINT), Nakhon Nayok Province, which is located $112 \mathrm{~km}$. away. Using a Colbalt-60 (Gammar Chamber 5000, Board of Radiation and Isotope Technology (BRIT), DAE, Mumbai, India), an irradiation dose of 50 Gy or 70 Gy for 45 seconds was applied by a qualified staff at TINT. After irradiation, part of the irradiated male pupae were transported back to the laboratory for further experiments, while most of them were transported to the field station at the City Center of 
Chachoengsao Province. Then small plastic containers holding irradiated pupae were put in plastic release cages prior to adult emergence, and a $10 \%$ sucrose solution was provided. After emergence, irradiated Wolbachia-infected males were double-checked for female contamination using a mouth aspirator to individually place male mosquitoes into the new plastic release cages. The number of mixed female mosquitoes from emerged pupae was recorded. The emerged irradiated Wolbachia-infected male mosquitoes, 1-3 days old, were then weekly released in the pilot trial to suppress Ae. aegypti mosquito vector populations at a village scale in Plaeng Yao District, Chachoengsao Province, Thailand. The number of released sterile male mosquitoes ranged from 9000 to 25,000 per week.

Testing sterility of irradiated Wolbachia-infected male and female mosquitoes

Preliminary experiments were set up to test the sterility of Ae. aegypti male and female mosquitoes after being exposed to an irradiation dose of 50 Gy or 70 Gy. In addition, during the 24-week open field trial, each lot of irradiated males and females was tested for sterility by mating them with non-irradiated females and non-irradiated males, respectively. In the experiments, the irradiated Wolbachia-infected male and female mosquitoes were separately introduced into cages sized $30 \mathrm{~cm} \times 30 \mathrm{~cm} \times$ $30 \mathrm{~cm}$, with a $10 \%$ sucrose solution provided. The non-irradiated Wolbachia-infected females were then introduced into the cage with irradiated Wolbachia-infected males, while the non-irradiated Wolbachia-infected males were introduced into the cage with irradiated Wolbachia-infected females. The ratio of irradiated Wolbachia-infected male and non-irradiated Wolbachia-infected female mosquitoes was 1:1. The same ratio was applicable with irradiated Wolbachia-infected female and nonirradiated Wolbachia-infected male mosquitoes. The mosquitoes were freely mated in the cages for 2-3 days. The females were then blood-fed using the Hemotek blood-feeding system (Hemotek Ltd., UK). Each bloodfeeding period lasted 1-2 hours and the Hemotek blood-feeding unit with new blood was re-introduced within 2-3 consecutive days. Blood-fed irradiated and non-irradiated Wolbachia-infected female mosquitoes were individually separated and placed in a plastic tube 7 $\mathrm{cm}^{3}$ in volume (diameter $3 \mathrm{~cm}$, height $5.5 \mathrm{~cm}$ ). Egg paper was placed over wet cotton inside each plastic tube for oviposition. After 3-4 days, the egg paper from each female mosquito was collected and the eggs were counted. Then it was dried and transferred into a glass container containing deionized water for hatching, as previously described. The number of hatched and un-hatched eggs from each individual female mosquito was recorded. The un-hatched eggs represented the sterility of the tested mosquitoes.

\section{Assessing survival and longevity of irradiated and non-} irradiated Wolbachia-infected male and female mosquitoes The emerged irradiated and non-irradiated Wolbachiainfected male and female mosquitoes were separately introduced into a cage sized $30 \mathrm{~cm} \times 30 \mathrm{~cm} \times 30 \mathrm{~cm}$, with a $10 \%$ sucrose solution provided. They were placed in the insectary at a temperature of $27 \pm 2{ }^{\circ} \mathrm{C}, 75 \pm 2 \%$ humidity and a photoperiod of L12:D12. The number of dead male and female mosquitoes was daily observed and recorded. The dead mosquitoes were then removed from the cage.

\section{Statistical analysis}

All statistical analyses were performed using SPSS 18.0 Mahidol University License (Chicago, SPSS Inc.). Weight, size, emergence, sex ratio of male and female pupae, sterility, and the longevity of irradiated Wolbachia-infected male and female mosquitoes were analyzed by means of one-way and two-way analyses of variance (ANOVA). Correlation between sex ratio and the number of released mosquitoes was analyzed by using Pearson's correlation.

\section{Results}

\section{Weight, size and emergence time of male and female pupae}

When comparing the weight of 1000 Wolbachia-infected Ae. aegypti male and female pupae, it was found that female pupae appeared to be heavier in weight, and this difference was statistically significant $(d f=2, F=74.940, P=0.001)$. The weight of female pupae ranged from $5.12-5.70 \mathrm{mg}$ $(5.49 \pm 0.32 \mathrm{mg})$, whereas those of the males ranged from $3.52-3.70 \mathrm{mg}$ (3.69 $\pm 0.16 \mathrm{mg})$ (Table 1).

When comparing the size of Wolbachia-infected Ae. aegypti male and female pupae, female pupae were significantly much bigger than male pupae in all parts, i.e., cephalothorax (female $=3.00 \pm 0.11 \mathrm{~mm}$ vs male $=$ $2.21 \pm 0.43 \mathrm{~mm}, t=-12.948, d f=59, P=0.000$ ), abdomen

Table 1 Comparison of average weight of Wolbachia-infected Aedes aegypti male and female pupae after being sex separated by using larval-pupal glass separators (Model 5412, John W. Hock Company, Gainesville, FL, USA)

\begin{tabular}{llllll}
\hline Sex & Rep. & $\begin{array}{l}N \\
(\text { Total })\end{array}$ & $\begin{array}{l}\text { Weight }(\mathrm{mg}) \\
(\text { Mean } \pm \text { SD) }\end{array}$ & $95 \% \mathrm{Cl}$ \\
\hline Male & 3 & 1000 & $3.69 \pm 0.16$ & $3.28-4.08$ & $0.001^{*}$ \\
Female & 3 & 1000 & $5.49 \pm 0.32$ & $4.69-6.30$
\end{tabular}

*Significant difference at $P<0.05$ 
$($ female $=3.13 \pm 0.42 \mathrm{~mm}$ vs male $=2.40 \pm 0.41 \mathrm{~mm}$, $t=-10.869, d f=59, P=0.000)$ and body length $($ female $=5.46 \pm 0.42 \mathrm{~mm} v s$ male $=4.08 \pm 0.74 \mathrm{~mm}$, $t=-12.714, d f=59, P=0.000$ ) (Table 2). Difference in the pupae size was an important parameter that was beneficial to mechanical sex separation.

When comparing emergence time between irradiated Wolbachia-infected male and female pupae, our results demonstrated that most male and female pupae emerged into adult mosquitoes on the second day after reaching the pupal stage, accounting for $74.60 \%$ and $62.05 \%$ respectively. A significant difference in emergence time was observed between male and female pupae $\left(d f=3, F_{\text {male }}=735.025, P=0.000 ; d f=3\right.$, $F_{\text {female }}=232.464, P=0.000$ ) (Table 3$)$. However, no significant difference was observed between the number of male and female pupae that emerged into adult mosquitoes $(t=-0.15, d f=11, P=0.989)$. It was observed that more than $98 \%$ of male and female pupae emerged into adults, which means that the irradiation dose of 70 Gy did not have negative effect on their emergence.

\section{Percentage of female contamination during pilot field release}

During the twenty-four weeks of the pilot field trial and the releases of sterile Ae aegypti males at the selected study site in Plaeng Yao District, Chachoengsao Province, each lot of sterile male pupae ranging from 1282 to $23,481$ (mean \pm SD $=5107.29 \pm 4,509.59)$ was inspected for female contamination. In total, 122,575 sterile male pupae were inspected for female contamination and the data are presented in Fig. 1 and Table 4. Results indicate that a total number of 60 females, ranging from 0 to 17, were found mixing with males during the mechanical sex

Table 2 Average size of Wolbachia-infected Aedes aegypti male and female pupae classified by cephalothorax, abdomen, and body length after being sex separated by using larval-pupal glass separators (Model 5412, John W. Hock Company, Gainesville, FL, USA)

\begin{tabular}{|c|c|c|c|c|c|c|}
\hline Morphology & Rep. & $\begin{array}{l}\text { Size }(\mathrm{mm} .) \\
(\text { Mean } \pm \mathrm{SD})\end{array}$ & $95 \% \mathrm{Cl}$ & $t$ & $d f$ & $P$ \\
\hline \multicolumn{7}{|c|}{ Cephalothorax } \\
\hline Male & 60 & $2.21 \pm 0.43$ & $-0.91--0.67$ & -12.948 & 59 & $0.0001^{*}$ \\
\hline Female & 60 & $3.00 \pm 0.11$ & & & & \\
\hline \multicolumn{7}{|l|}{ Abdomen } \\
\hline Male & 60 & $2.40 \pm 0.41$ & $-0.86--0.59$ & -10.869 & 59 & $0.0001^{*}$ \\
\hline Female & 60 & $3.13 \pm 0.42$ & & & & \\
\hline \multicolumn{7}{|l|}{ Body length } \\
\hline Male & 60 & $4.08 \pm 0.74$ & $-1.60--1.17$ & -12.714 & 59 & $0.0001^{*}$ \\
\hline Female & 60 & $5.46 \pm 0.42$ & & & & \\
\hline
\end{tabular}

*Significant difference at $P<0.05$ separation process (mean $\pm \mathrm{SD}=2.50 \pm 4.17$ ) (Table 4), accounting for $0.06 \pm 0.10 \%$ female contamination. Remarkably, when 1282 to 5,000 sterile males were inspected during the first twelve weeks of the intervention, the female contamination was $0.10 \pm 0.13 \%(2.40 \pm 3.42)$. However, there were lower numbers of female contamination during the second twelve weeks of intervention, i.e. $0.02 \pm 0.02 \%$ (2.67 \pm 5.43$)$, even though samples containing more than 5000 sterile males were inspected, and a statistically significant difference was observed between the two groups of intervention ( $t=2.317, d f=11, P=0.041)$ (Table 4$)$. It is worth noting that when the largest sample was applied for sex separation $(23,481$ male pupae), the female contamination was $0.07 \%$. When compared between the first and second 12-week periods, it was noticed that, there was a fluctuation in the percentage of female contamination during the first twelve weeks of release, but this was relatively small, accounting for $0.02-0.32 \%$. This percentage of female contamination was remarkably reduced in the second twelve weeks of intervention. In conclusion, at least $99 \%$ of sterile males were purely separated from females, demonstrating high efficiency in the manual sex separation process during this pilot intervention.

\section{Sterility of irradiated Wolbachia-infected male and female mosquitoes}

A preliminary study showed that an irradiation dose of 50 Gy was sufficient to induce complete sterility in Ae. aegypti females but not in males (Table 5). Wolbachia-infected males irradiated ( $\widehat{\jmath}$ ir-w) at 50 Gy could still produce viable eggs when mated with non-irradiated Wolbachia-infected females $(q \mathrm{nr}-\mathrm{w})$. The average percentage of eggs hatched into the first-instar larvae was $8 \%$, while the egg hatch rate was zero when irradiated Wolbachia-infected females ( $Q$ ir-w) mated with non-irradiated Wolbachia-infected males (ठ̋nr-w). No eggs were hatched when Wolbachia-infected males and females were irradiated ( $\mathrm{ir}-\mathrm{w} \&$ ir-w) at $70 \mathrm{~Gy}$ and then were mated with non-irradiated Wolbachia-infected females and males ( + nr-w \& $\$ nr-w) respectively.

Results of mating tests performed during the 24-week open field trial between irradiated and non-irradiated Wolbachia-infected Ae. aegypti male and female mosquitoes are shown in Fig. 2 and Table 6. When irradiated Wolbachia-infected males mated with non-irradiated Wolbachia-infected female mosquitoes, the females could still lay eggs (mean total eggs $=1,341.13 \pm 431.61$ ), but the number of hatched eggs was quite low (mean hatched eggs $=1.04 \pm 2.18$ ) (Fig. 2, Table 6), which demonstrates that the irradiated Wolbachia-infected males were highly sterile and that their sterility could provoke nearly complete sterility through reduction of the egg hatch rate in the next generation (mean hatch rate $=0.07 \pm 0.13$ ). Irradiated Wolbachiainfected female mosquitoes seemed to be more sensitive to 
Table 3 Average number of emerged Wolbachia-infected Aedes aegypti male and female pupae after being irradiated at 70 Gy

\begin{tabular}{|c|c|c|c|c|c|c|c|c|c|}
\hline Sex & Rep. & $\begin{array}{l}\text { N } \\
\text { (Total no.) }\end{array}$ & Day & $\begin{array}{l}\text { Average emerged } \\
\text { mosquitoes } \\
\text { (Mean } \pm \text { SD) }\end{array}$ & $\begin{array}{l}\text { Average non-emerged } \\
\text { mosquitoes }\end{array}$ & $\begin{array}{l}\% \text { emerged } \\
\text { mosquitoes }\end{array}$ & $95 \% \mathrm{Cl}$ & $F$ & $P$ \\
\hline \multirow[t]{4}{*}{ Male } & 3 & 1500 & 1 & $41.67 \pm 12.86$ & $0.00 \pm 0.00$ & $8.33 \pm 2.57$ & $1.95-14.72$ & 735.025 & $0.00^{*}$ \\
\hline & & & 2 & $369.00 \pm 14.73$ & $0.00 \pm 0.00$ & $73.80 \pm 2.95$ & $66.48-81.12$ & & \\
\hline & & & 3 & $80.67 \pm 7.77$ & $0.00 \pm 0.00$ & $16.13 \pm 1.55$ & $12.27-19.99$ & & \\
\hline & & & 4 & $3.33 \pm 3.21$ & $5.33 \pm 4.04$ & $0.67 \pm 0.64$ & $-0.93-2.26$ & & \\
\hline \multirow[t]{4}{*}{ Female } & 3 & 1500 & 1 & $13.33 \pm 7.09$ & $0.00 \pm 0.00$ & $2.67 \pm 1.42$ & $-0.86-6.19$ & 232.464 & $0.00^{*}$ \\
\hline & & & 2 & $306.33 \pm 23.44$ & $0.00 \pm 0.00$ & $61.27 \pm 4.69$ & $49.62-72.91$ & & \\
\hline & & & 3 & $168.00 \pm 21.28$ & $0.00 \pm 0.00$ & $33.60 \pm 4.26$ & $23.03-44.17$ & & \\
\hline & & & 4 & $6.00 \pm 1.73$ & $6.33 \pm 1.15$ & $1.20 \pm 0.35$ & $0.34-2.06$ & & \\
\hline
\end{tabular}

*Significant difference at $P<0.05$

50 Gy and 70 Gy irradiation doses, since they lost their ability to lay eggs (mean total eggs $=0.00 \pm 0.00$ ) or they were completely sterile when mated with non-irradiated Wolbachia-infected males (mean hatch rate $=0.00 \pm 0.00$ ) (Tables 5 and 6).

When comparing the crosses between non-irradiated Wolbachia-infected females mated with irradiated Wolbachia-infected males ( $\mathrm{nr}-\mathrm{w}$ female $\times$ ir-w male) and irradiated Wolbachia-infected females mated with non-irradiated Wolbachia-infected males (ir-w female $\times \mathrm{nr}-\mathrm{w}$ male), there were statistically significant differences in the total number of eggs $(t=15.22, d f=23, P=0.000)$, the hatched eggs $(t=2.35, d f=23, P=0.028)$ and the egg hatch rate $(t=2.60, d f=23, P=0.016)$ (Table 6). In conclusion, an irradiation dose of 70 Gy induces high to nearly complete sterility in male mosquitoes. Non-irradiated Wolbachia-infected females that mated with irradiated Wolbachia-infected males could still lay eggs (mean total eggs $=1,341.13 \pm 431.61$ eggs), but the number of hatched eggs was quite low (mean hatched eggs $=1.04 \pm 2.18$ ), which demonstrated that the irradiated Wolbachia-infected male mosquitoes were highly sterile. Contrary to males, irradiated Wolbachia-infected females with a dose of 70 Gy either lost their ability to lay eggs (mean total eggs $=0.00 \pm 0.00$ eggs) after mating with non-irradiated Wolbachia-infected males or were completely sterile (mean hatch rate $=0.00 \pm 0.00$ ) (Table 6). Therefore, since the males and females used in the crosses were both Wolbachia-infected, sterility should be induced by irradiation, with a dose of 70 Gy being the optimum one for Wolbachia-infected Ae. aegypti male mosquitoes, as it could make them fully sterile. Moreover, in the case where irradiated Wolbachiainfected females were accidentally released, these females could not reproduce because they were fully sterile due to the effect of irradiation.

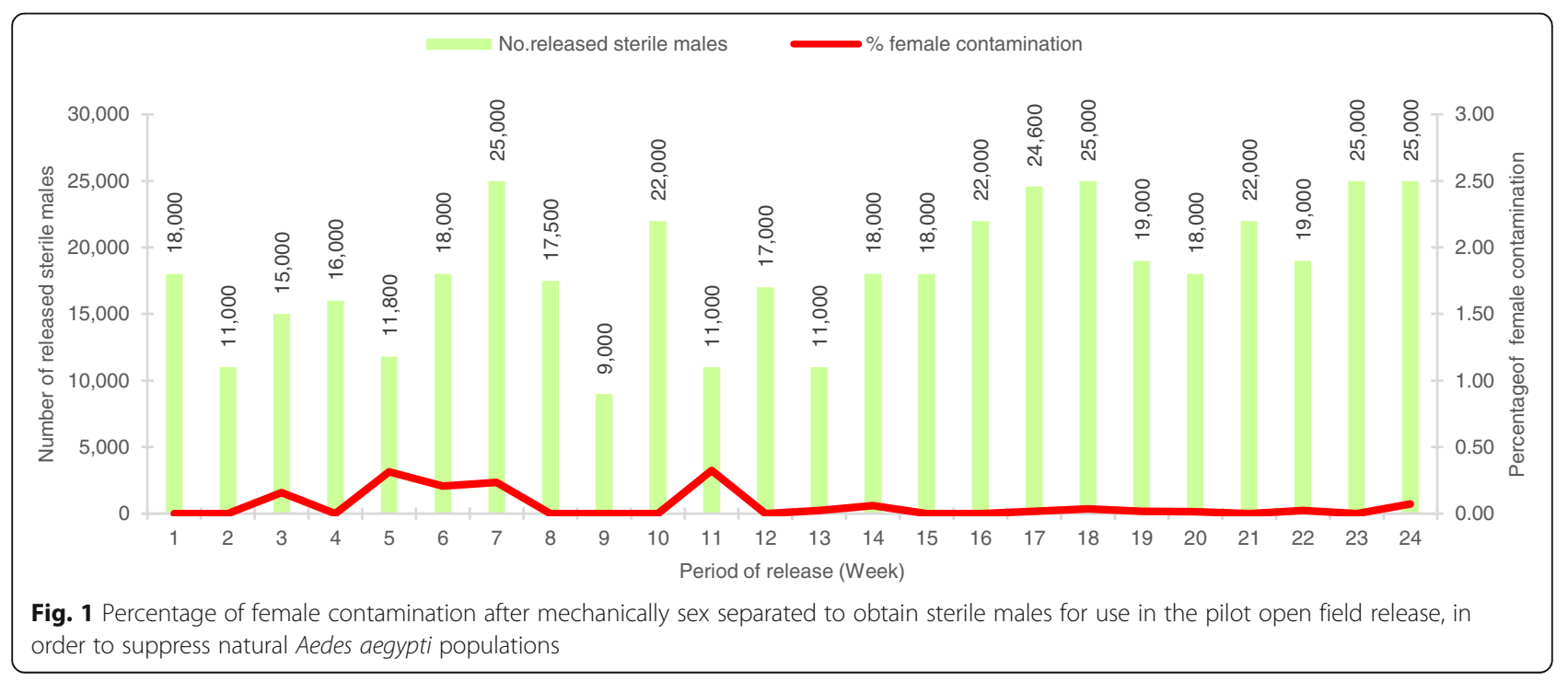


Table 4 Percentage of female contamination after mechanical sex separation, comparing the first and the second twelve weeks of intervention

\begin{tabular}{lllllll}
\hline Replicate & $\begin{array}{l}\text { No. sampling } \\
\text { sterile males }\end{array}$ & $\begin{array}{l}\% \text { female } \\
\text { contamination } \\
\text { (Mean } \pm \text { SD) }\end{array}$ & $95 \% \mathrm{Cl}$ & df & $t$ & $P$ \\
\hline 12 & 38,253 & $0.11 \pm 0.13$ & $0.004-0.169$ & 11 & 2.317 & $0.041^{*}$ \\
12 & 84,322 & $0.02 \pm 0.02$ & & & & \\
\hline
\end{tabular}

${ }^{*}$ significant difference at $P<0.05$

\section{Longevity and survival rate of irradiated and non- irradiated Wolbachia-infected males and females following sex separation}

Overall, adult longevity in irradiated Wolbachia-infected Ae. aegypti mosquitoes varied from 3 to 44 days, while for non-irradiated Wolbachia-infected Ae. aegypti, it was from 2 to 71 days. The differences in longevity and survival rate between male and female mosquitoes were mostly observed from 12 to 35 days.

For irradiated Wolbachia-infected mosquitoes, males seemed to live longer and had a higher survival rate when compared to females (longevity: đo ir-w $=22.14 \pm 11.44$ days $v s \quad+\mathrm{ir}-\mathrm{w}=18.47 \pm 9.81$ days; survival rate: Jir-w $=0.63 \pm 0.26$ vs ir $-\mathrm{w}=0.55 \pm 0.32$ ), and the differences were statistically significant $(t=5.962, d f=34$, $P=0.000$ ) (Fig. 3, Table 7). On the contrary, the non-irradiated Wolbachia-infected females lived longer and had a higher survival rate, when compared to the non-irradiated Wolbachia-infected males (longevity: $q_{\mathrm{nr}-\mathrm{w}}=29.64 \pm 1.03$ days $v s$ ônr-w $=23.31 \pm 0.91$ days; survival rate: ${ }^{\circ} \mathrm{nr}-\mathrm{w}=0.76 \pm 0.25$ vs $\widehat{o} \mathrm{nr}-\mathrm{w}=0.51 \pm 0.34$ ), and the differences were statistically significant $(t=-10.687$, $d f=43, P=0.000)$.

However, when comparing either males or females between irradiated and non-irradiated, we found no difference in longevity and survival rate between the irradiated Wolbachia-infected males and the non-irradiated Wolbachia-infected males (longevity: ${ }^{1} \mathrm{ir}-\mathrm{w}=23.77 \pm 12.11$ days $v s \quad \hat{n} \mathrm{n}-\mathrm{w}=23.31 \pm 0.91$ days; survival rate: ठैir-w $=0.52 \pm 0.32 v s$ ônr-w $=0.51 \pm 0.34, t=0.795$, $d f=43, P=0.431)$. On the contrary, the irradiated Wolbachia-infected females have a much shorter lifespan and low survival rate when compared to the non-irradiated Wolbachia-infected females (longevity: O $\mathrm{ir}-\mathrm{w}=18.47 \pm 9.81$ days $v s$ . $\mathrm{nr}-\mathrm{w}=25.44 \pm 1.07$ days; survival rate: $\uparrow \mathrm{ir}-\mathrm{w}=0.55 \pm 0.32 v s+\mathrm{nr}-\mathrm{w}=0.85 \pm 0.19$ ), and the differences were statistically significant $(t=-8.738$, $d f=34, P=0.000)$.

\section{Discussion}

Size difference in male and female pupae was the basis for sex separation, especially for mass production of sterile males to be used in the SIT programmes. Various factors such as larval density, diet, temperature and others affect pupae size, and a standardized rearing condition was required in order to effectively separate males from females $[15,19]$. In our study, female pupae appeared to be significantly larger than males and could easily be separated by mechanical tools, indicating our appropriate rearing condition. Importance of male size has previously been highlighted in the mating success, larger males having a greater mating capacity than smaller males [21-24].

Sex separation at the pupal stage was more convenient and practical when compared to adults [25]. Moreover, late pupae were more tolerant to the irradiation process than early ones, in terms of an effect on adult emergence and mortality. In our study, the larval-pupal glass separators were used in sex separation, and a high survival rate was observed. More than $62 \%$ and $74 \%$ of female and male pupae respectively emerged on the second day. Therefore, it was more practical and recommended to sex separate them on the second day of pupation, when there was still a high percentage of males, as indicated in Medici et al. (2011) [26]. Some studies reported using metal sieves to separate male from female pupae with a high purity, but only 15 - $25 \%$ of males were recovered [15].

In the past sterile male release programmes, only less than $5 \%$ of female contamination was acceptable, but currently this is considered as unacceptable [12]. In this

Table 5 Sterility of irradiated Wolbachia-infected male and female Aedes aegypti mosquitoes after being exposed to the irradiation dosages of 50 Gy or 70 Gy

\begin{tabular}{|c|c|c|c|c|c|c|}
\hline Experiment & $\begin{array}{l}\text { No. of } \\
\text { females (F0) }\end{array}$ & $\begin{array}{l}\text { No. of egg-laid } \\
\text { females (FO) }\end{array}$ & $\begin{array}{l}\text { Total no. } \\
\text { of eggs }\end{array}$ & Eggs/female & $\begin{array}{l}\text { No. of hatched } \\
\text { eggs }\end{array}$ & $\begin{array}{l}\text { Egg hatch } \\
\text { rate }\end{array}$ \\
\hline \multicolumn{7}{|l|}{ Radiation dosage } \\
\hline \multicolumn{7}{|l|}{50 Gy } \\
\hline$\delta \mathrm{ir}-\mathrm{w} \times \times q \mathrm{nr}-\mathrm{w}$ & 27 & 23 & 1,021 & 44.39 & 80 & 0.08 \\
\hline$\hat{o} \mathrm{nr}-\mathrm{w} \times q$ ir-w & 27 & 0 & 0 & 0 & 0 & 0 \\
\hline \multicolumn{7}{|l|}{70 Gy } \\
\hline$\widehat{o} \mathrm{ir}-\mathrm{w} \times q \mathrm{nr}-\mathrm{w}$ & 18 & 10 & 404 & 40.40 & 0 & 0 \\
\hline$\hat{o} \mathrm{nr}-\mathrm{w} \times q$ ir-w & 27 & 0 & 0 & 0 & 0 & 0 \\
\hline
\end{tabular}




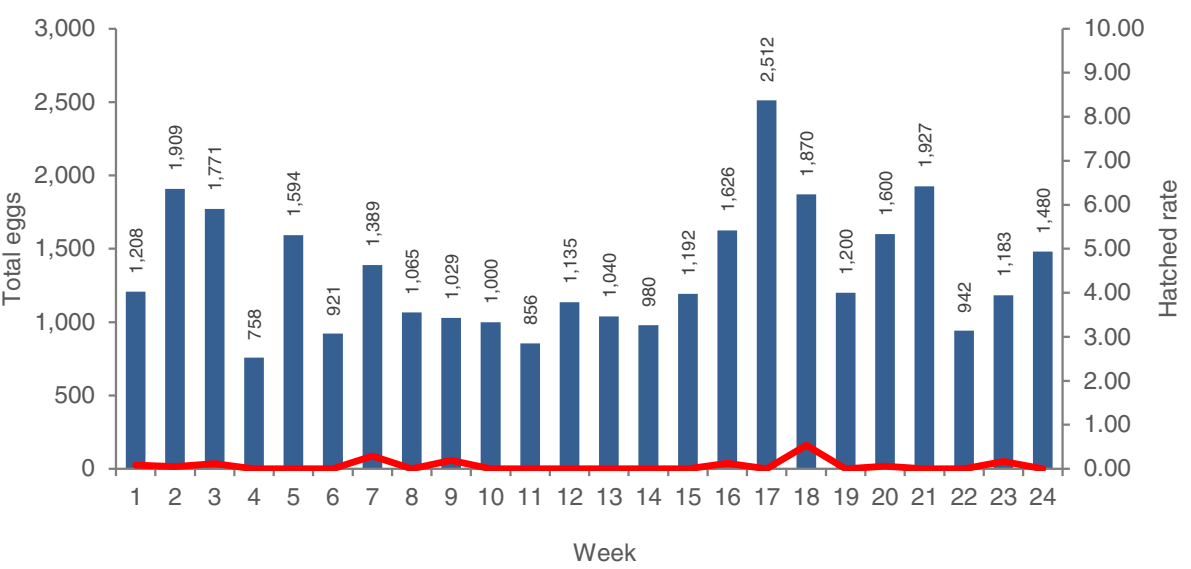

Fig. 2 Total number of eggs and egg hatch rate from mating between irradiated Wolbachia-infected males and non-irradiated Wolbachiainfected females of Aedes aegypti mosquitoes

study, at least $99 \%$ of male pupae were successfully separated, and more than $98 \%$ of males recovered, after being irradiated. Therefore, sex separation at the pupal stage by using adjustable glass plates could be an appropriate method to use for small-scale pilot trials. However, care should be taken in terms of maintaining harmonized adjustment at the early stage of sex separation. Skill of the personnel working with manual sex separation using larval-pupal glass separators was important to obtain a high percentage of male pupae and minimal female contamination. Our pilot intervention showed a fluctuation in the percentage of female contamination during the first twelve weeks, but not in the second period when the personnel were more skillful and more familiar with the technique.

Different mosquito species require different irradiation dose to achieve complete sterility. In our experiments, a difference in susceptibility to irradiation was observed between the male and female Ae. aegypti mosquitoes, as reported in other insects [3, 4]. Females were more susceptible to irradiation than males, being completely sterile at a lower dose. When no perfect sex separation method is available, female mosquitoes could accidentally be released together with males. Therefore, it is necessary to consider an irradiation dose that could fully sterilize both male and female mosquitoes, in order to eliminate the risk of releasing fertile mosquitoes. In our case, any released females not only would be sterile but also would exhibit reduced risk of pathogen transmission, since they were infected with Wolbachia [27].

In a system of SIT/IIT, the sterility of released males would be due to both Wolbachia and low-dose irradiation, while the Wolbachia-infected female sterility would only be caused by irradiation. In our preliminary study, irradiation doses at both 50 Gy and 70 Gy could induce complete sterility in Wolbachia-infected Ae. aegypti females; but at $50 \mathrm{~Gy}$, only female but not male mosquitoes were completely sterile. Non-irradiated Wolbachia-infected females that mate with irradiated Wolbachia-infected males still produced hatched eggs, although at very low egg hatch

Table 6 Analysis of variance of total number of eggs, hatched eggs and egg hatch rate between irradiated Wolbachia-infected (ふ̋ir-w) males and non-irradiated Wolbachia-infected (qnr-w) females vs non-irradiated Wolbachia-infected (ठ̊nr-w) males and irradiated Wolbachia-infected (Oir-w) females of Aedes aegypti mosquitoes

\begin{tabular}{|c|c|c|c|c|c|c|c|}
\hline Mating pair & Rep. & $N$ & Mean \pm SD & $95 \% \mathrm{Cl}$ & $t$ & $d f$ & $P$ \\
\hline \multicolumn{8}{|l|}{ Total eggs } \\
\hline đir-w $\times$ O nnr-w & 24 & 1110 & $1341.13 \pm 431.61$ & $1158.87-1523.38$ & 15.22 & 23 & $0.0001^{*}$ \\
\hline$\delta \mathrm{nr}-\mathrm{w} \times$ Q irr-w & 24 & 1105 & $0.00 \pm 0.00$ & & & & \\
\hline \multicolumn{8}{|l|}{ Hatched eggs } \\
\hline$\widehat{\partial} \mathrm{ir}-\mathrm{w} \times \stackrel{+}{\mathrm{nnr}-\mathrm{w}}$ & 24 & 1110 & $1.04 \pm 2.18$ & $0.12-1.96$ & 2.35 & 23 & $0.028^{*}$ \\
\hline ônr-w x P ir-w & 24 & 1105 & $0.00 \pm 0.00$ & & & & \\
\hline \multicolumn{8}{|l|}{ Egg hatch rate } \\
\hline$\delta$ orr-w $\times$ onr-w & 24 & 1110 & $0.07 \pm 0.13$ & $0.01-0.12$ & 2.60 & 23 & $0.016^{*}$ \\
\hline ônr-w x O ir-w & 24 & 1105 & $0.00 \pm 0.00$ & & & & \\
\hline
\end{tabular}

*Significant difference at $P<0.05$ 


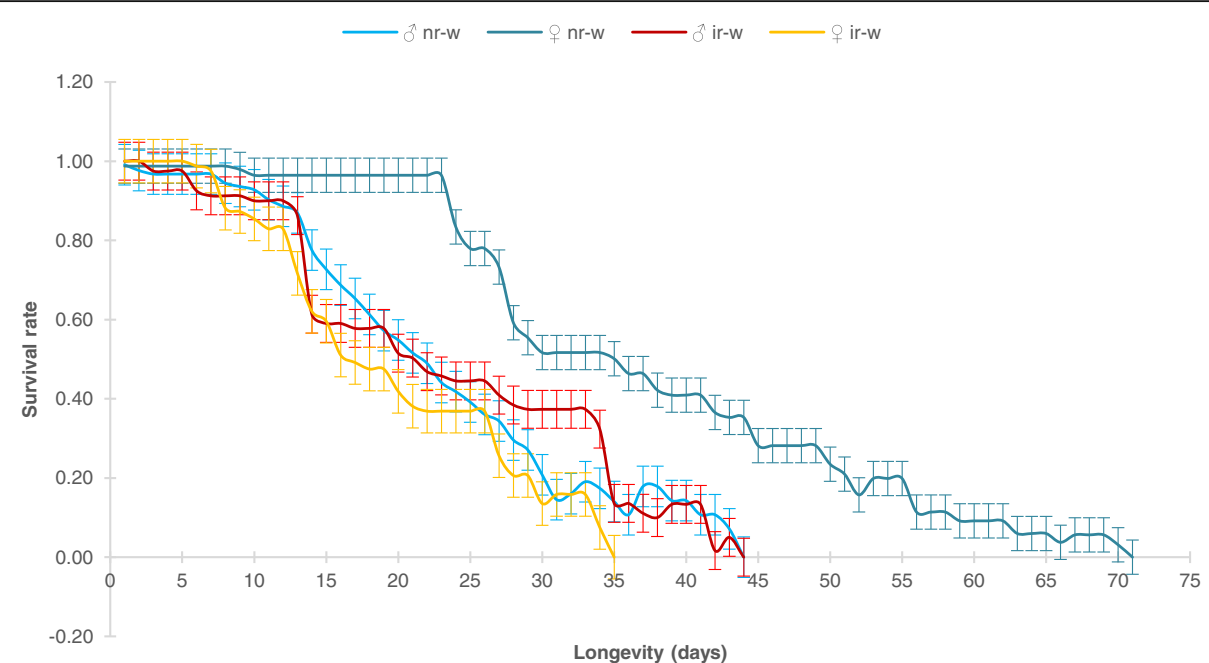

Fig. 3 Mean longevity and survival rate of non-irradiated and irradiated Wolbachia-infected Aedes aegypti male and female mosquitoes, after being sex-separated by using larval-pupal glass separators

rate of 0.08 . The sterility induced in these females was due to irradiation, as both males and females were Wolbachia-infected and our previous work indicated incomplete CI when non-irradiated Wolbachia-uninfected females were mated with non-irradiated Wolbachia-infected males [18]. But for the irradiated Wolbachia-infected females, this dosage induced complete sterility and no egg production was observed. For the quality control test of all 24 lots of mosquitoes which were irradiated at $70 \mathrm{~Gy}$, we found a very low egg hatch rate of 0.07 of all crosses between irradiated Wolbachia-infected males and non-radiated Wolbachia-infected females. Hence, irradiation at $70 \mathrm{~Gy}$ was considered the practical dose to induce sterility in Wolbachia-infected Ae aegypti male mosquitoes for being released in our pilot field suppression trial. Based on the above, the combined SIT/IIT offers a safe and biosecure approach for population suppression programmes against Ae. aegypti similar to the one recently developed and applied against Aedes albopictus [8-10].
In terms of longevity, female mosquitoes were reported to live up to 90 days [28] or, in some cases, up to 150 days $[29,30]$. Our results showed a significant impact of irradiation on female longevity and survival rate. The longevity of irradiated Wolbachia-infected Ae. aegypti females was reduced to nearly 18 days on average. In the case of males, our results showed almost no effect on longevity and survival rate, since no significant differences were observed between irradiated and non-irradiated Wolbachia-infected Ae. aegypti males.

In our study, we found that irradiated Wolbachia-infected males lived slightly longer than irradiated Wolbachia-infected females. However, our results contradict other studies [28, 31] that reported longer lifespans among females than males, whether both sexes were reared separately or together. The difference in longevity between males and females demonstrated in our study is related to the fact that females are more radiosensitive than males. Radiation has been shown to decrease adult

Table 7 Analysis of variance of longevity and survival rate between irradiated Wolbachia-infected (ir-w) and non-irradiated Wolbachiainfected (nr-w) Aedes aegypti male and female mosquitoes, after being sex-separated by using larval-pupal glass separators

\begin{tabular}{|c|c|c|c|c|c|c|c|}
\hline Experiment & $N$ & $\begin{array}{l}\text { Longevity (day) } \\
\text { (Mean } \pm \text { SD) }\end{array}$ & Survival rate & $95 \% \mathrm{Cl}$ & $t$ & $d f$ & $P$ \\
\hline o ir-w & 120 & $21.11 \pm 10.14$ & $0.63 \pm 0.26$ & $0.06-0.11$ & 5.962 & 34 & $0.0001^{*}$ \\
\hline q ir-w & 120 & $18.47 \pm 9.81$ & $0.55 \pm 0.32$ & & & & \\
\hline ônr-w & 120 & $23.31 \pm 0.91$ & $0.51 \pm 0.34$ & $-0.29--0.20$ & -10.687 & 43 & $0.0001^{*}$ \\
\hline q nr-w & 120 & $29.64 \pm 1.03$ & $0.76 \pm 0.25$ & & & & \\
\hline$\delta \mathrm{ir}-\mathrm{w}$ & 120 & $23.77 \pm 12.11$ & $0.52 \pm 0.32$ & $-0.02-0.04$ & 0.795 & 43 & 0.431 \\
\hline$\partial \mathrm{nr}-\mathrm{w}$ & 120 & $23.31 \pm 0.91$ & $0.51 \pm 0.34$ & & & & \\
\hline q ir-w & 120 & $18.47 \pm 9.81$ & $0.55 \pm 0.32$ & $-0.37--0.23$ & -8.738 & 34 & $0.0001^{*}$ \\
\hline q nr-w & 120 & $25.44 \pm 1.07$ & $0.85 \pm 0.19$ & & & & \\
\hline
\end{tabular}

*Significant difference at $P<0$ 
life span, including its subsequent generations [21, 32]. Moreover, changes in hatchability, followed by adult emergence and longevity, were more prominently observed with increasing irradiation dose [32]. At the pupal stage, irradiation can negatively affect adult emergence and consequently survival rates [8]. In this study, irradiated Wolbachia-infected females exhibited higher mortality when compared to irradiated Wolbachia-infected males when they were exposed at the same irradiation dose. However, irradiated Wolbachia-infected females in our study were only fed with a sucrose solution and no blood meals were provided. This could be one of the important parameters to explain for the shorter lifespan of these females. Other studies have shown that Ae. aegypti females fed only on a sucrose solution had a shorter lifespan that those fed with either blood alone or both blood and sugar [33-35]. This observation was most likely due to a depletion of protein reserves [33].

\section{Conclusions}

In conclusion, application of SIT requires various components, including a mass-rearing process that consists of many important parameters, such as diet, rearing conditions, and importantly sex separation. Our study provides useful information in terms of the practical application of a mechanical sex separation method to obtain only males for further sterilization and open small-scale field release. However, this approach would not be effective for a very large scale application. There is certainly an urgent need for further research to develop novel, efficient and cost-effective sex separation techniques to support large scale SIT applications $[13,36]$. We also demonstrated the quality of male and female mosquitoes, in terms of survival and longevity after sex separation and sterilization, which would be beneficial for planning a field release in order to suppress natural populations of Ae. aegypti mosquitoes. In our studies, we found no significant difference between non-radiated and irradiated males in term of survival and longevity while irradiated females had shorter life span. In addition, both irradiated males and females were completely sterile when they were irradiated at 70 Gy. The overall quality including the male mating competitiveness of the released irradiated Wolbachia-infected Ae. aegypti mosquitoes needs to be further investigated particularly because this is a critical factor for the successful field implementation of the combined SIT/IIT approach.

\footnotetext{
Acknowledgement

The authors would like to thank the staff of the Center of Excellence for Vectors and Vector-Borne Diseases, Faculty of Science, Mahidol University, i.e., Natchaya Klinpikul, Thitikarn Summat and Kuang Chalongpak for lab assistance; Kitti Thienthong and Pitak Promchareon for field assistance; Thodsapon Thannarin for assisting in irradiation process, David A. Blyler for English editing, and lastly the editor, Kostas Bourtzis, and anonymous reviewers for critically reviewing and improving the quality of the manuscript. This study has benefit from discussions
}

at the International Atomic Energy Agency (IAEA)-funded meetings for the Coordinated Research Project "Exploring genetic, molecular, mechanical and behavioural methods of sex separation in mosquitoes".

\section{Funding}

This work was financially supported by Mahidol University and the International Atomic Energy Agency (IAEA). Publication costs for this study was provided by the International Atomic Energy Agency as part of the Coordinated Research Project "Exploring genetic, molecular, mechanical and behavioural methods of sex separation in mosquitoes".

\section{Availability of data and materials}

All data and materials are available from the corresponding author upon request.

\section{About this supplement}

This article has been published as part of Parasites \& Vectors Volume 11 Supplement 2, 2018: Exploring genetic molecular, mechanical and behavioural methods of sex separation in mosquitoes. The full contents of the supplement are available online at https://parasitesandvectors.biomedcentral.com/ articles/supplements/volume-11-supplement-2.

\section{Authors' contributions}

PK designed and supervised lab and field experiments, and wrote the manuscript. NK conducted laboratory experiments. SN conducted statistical analysis and wrote the manuscript. WL assisted in radiation process. All authors read and approved the final manuscript.

\section{Ethics approval and consent to participate}

The pilot suppression trial of Aedes aegypti using a combined sterile insect technique and Wolbachia-based approach was reviewed and approved by Mahidol University Institutional Review Board (MU-CIRB 2016/085.0407).

\section{Consent for publication}

Not applicable.

\section{Competing interests}

The authors declare that they have no competing interests.

\section{Publisher's Note}

Springer Nature remains neutral with regard to jurisdictional claims in published maps and institutional affiliations.

\section{Author details}

${ }^{1}$ Center of Excellence for Vectors and Vector-Borne Diseases, Faculty of Science, Mahidol University at Salaya, Nakhon Pathom 73170, Thailand. ${ }^{2}$ Department of Biology, Faculty of Science, Mahidol University, Bangkok 10400, Thailand. ${ }^{3}$ Thailand Institute of Nuclear Technology, Ministry of Science and Technology, Nakhon Nayok 26120, Thailand.

\section{Published: 24 December 2018}

\section{References}

1. Lees RS, Gilles JRL, Hendrichs J, Vreysen MJB, Bourtzis K. Back to the future: the sterile insect technique against mosquito disease vectors. Opin Insect Sci. 2015;10:156-62.

2. Saridaki A, Bourtzis K. Wolbachia: More than just a bug in insects genitals. Curr Opin Microbiol. 2010;13(1):67-72

3. Nikolouli K, Colinet H, Renault D, Enriquez T, Mouton L, Gibert P. Sterile insect technique and Wolbachia symbiosis as potential tools for the control of the invasive species Drosophila suzukii. J Pest Sci. 2018;91(2):489-503.

4. Bourtzis K, Robinson AS. Insect pest control using Wolbachia and radiation. In: Bourtzis K, Miller T, editors. Insect Symbiosis 2. Boca Raton, FL, USA: Taylor and Francis, CRC Press; 2006. p. 225-46.

5. Bourtzis K, Dobson SL, Xi Z, Rasgon JL, Calvitti M, Moreira LA, et al. Harnessing mosquito-Wolbachia symbiosis for vector and disease control. Acta Trop. 2014;132:S150-63.

6. Bourtzis K, Lees RS, Hendrichs J, Vreysen MJB. More than one rabbit out of the hat: radiation, transgenic and symbiont-based approaches for sustainable management of mosquito and tsetse fly populations. Acta Trop. 2016;157:115-30. 
7. Brelsfoard CL, Clair WS, Dobson SL. Integration of irradiation with cytoplasmic incompatibility to facilitate a lymphatic filariasis vector elimination approach. Parasit Vectors. 2009;2(1):38.

8. Zhang D, Zheng X, Xi Z, Bourtzis K, Gilles JR. Combining the sterile insect technique with the incompatible insect technique: I. Impact of Wolbachia infection on the fitness of triple and double-infected strains of Aedes albopictus. PLoS One. 2015a;10:e0121126.

9. Zhang D, Lees RS, Xi Z, Gilles JR, Bourtzis K. Combining the sterile insect technique with Wolbachia-based approaches: II. A safer approach to Aedes albopictus population suppression programmes, designed to minimize the consequences of inadvertent female release. PLoS One. 2015b;10:e0135194.

10. Zhang D, Lees RS, Xi Z, Bourtzis K, Gilles JR. Combining the sterile insect technique with the incompatible insect technique: III- Robust mating competitiveness of irradiated triple Wolbachia-infected Aedes albopictus males under semi-field conditions. PLoS One. 2016;11:e0151864.

11. Bellini R, Calvitti M, Medici A, Carrieri M, Celli G, Maini S. Use of the Sterile Insect Technique against Aedes albopictus in Italy: first results of a pilot trial. In: Area-Wide Control of Insect Pests. Dordrecht: Springer; 2007. p. 505-15.

12. Sharma VP, Patterson RS, Ford HR. A device for the rapid separation of male and female mosquito pupae. Bull WHO. 1972;47(3):429-43.

13. Gilles JRL, Schetelig MF, Scolari F, Marec F, Capurro ML, Franz G, et al. Towards mosquito sterile insect technique programmes: exploring genetic, molecular, mechanical and behavioural methods of sex separation in mosquitoes. Acta Trop. 2014;132:S178-87.

14. Benedict MQ, Knols BGJ, Bossin HC, Howell PI, Mialhe E, Caceres C, et al. Colonisation and mass rearing: learning from others. Malaria J. 2009;8(Suppl 2):S4.

15. Papathanos PA, Bossin HC, Benedict MQ, Catteruccia F, Malcolm CA, Alphey $L$, et al. Sex separation strategies: past experience and new approaches. Malaria J. 2009;8(2):S2-5.

16. Balestrino F, Puggioli A, Carrieri M, Bouyer J, Bellini R. Quality control methods for Aedes albopictus sterile male production. PLoS Negl Trop Dis. 2017;11(9):0005881.

17. Ernawan B, Tambunan USF, Sugoro I, Sasmita HI. Effects of gamma irradiation dose-rate on sterile male Aedes aegypti. AIP Conf Proc. 2017; 1854:020010.

18. Ruang-areerate T, Kittayapong P. Wolbachia transinfection in Aedes aegypt: A potential gene driver of dengue vectors. Proc Nat Acad Sci (PNAS). USA. 2006;103:12534-9.

19. Mikery O, Serrano K, Sanchez D, del Pilar MCM. Efficiency of the separation of Aedes (Stegomyia) albopictus (Diptera: Culicidae) male and female pupae using a sieving device. Acta Zool Mex. 2015;31(1):113-5.

20. Timmermann SE, Briegel $\mathrm{H}$. Larval growth and biosynthesis of reserves in mosquitoes. J Insect Physiol. 1999;45(5):461-70.

21. Helinski MEH, Harrington LC. Male mating history and body size influence female fecundity and longevity of the dengue vector Aedes aegypti. J Med Entomol. 2011;48(2):202-11.

22. Bellini R, Medici A, Puggioli A, Balestrino F, Carrieri M. Pilot field trials with Aedes albopictus, irradiated sterile males in Italian urban areas. J Med Entomol. 2013;50(2):317-25.

23. Gary RE, Cannon JW, Foster WA. Effect of sugar on male Anopheles gambiae mating performance, as modified by temperature, space, and body size. Parasit Vectors. 2009;2:19.

24. Ponlawat $A$, Harrington LC. Factors associated with male mating success of the dengue vector mosquito, Aedes aegypti. Am J Trop Med Hyg. 2009;80: 395-400.

25. Akter $\mathrm{H}$, Khan SA. Sensitivity of immature stages of dengue causing mosquito, Aedes aegypti (L.) to Gamma radiation. J Entomol. 2014;11(2):56-67.

26. Medici A, Carrieri M, Scholte EJ, Maccagnani B, Dindo ML, Bellini R. Studies on Aedes albopictus larval mass-rearing optimization. J Econ Entomol. 2011; 104(1):266-73.

27. Moreira LA, Iturbe-Ormaytxe I, Jeffery JA, Lu G, Pyke AT, Hedges LM, et al. A Wolbachia symbiont in Aedes aegypti limits infection with dengue, chikungunya and Plasmodium. Cell. 2009;139:1268-78.

28. Maciel-De-Freitas R, Codeço CT, Lourenço-De-Oliveira R. Body sizeassociated survival and dispersal rates of Aedes aegypti in Rio de Janeiro. Med Vet Entomol. 2007;21(3):84-292.

29. Chadee DD, Martinez R, Sutherland JM. Aedes aegypti (L.) mosquitoes in Trinidad, West Indies: longevity case studies. J Vect Ecol. 2017;42(1):130-5.

30. Brady OJ, Johansson MA, Guerra CA, Bhatt S, Golding N, Pigott DM, et al. Modelling adult Aedes aegypti and Aedes albopictus survival at different temperatures in laboratory and field settings. Parasit Vectors. 2013;6(1):351.
31. Liles JN, Delong DM. The longevity and productivity of adult male and female Aedes aegypti when reared separately and together on three different diets. Ann Entomol Soc Am. 1960;53(2):277-80.

32. Shetty V, Shetty NJ, Harini BP, Ananthanarayana SR, Jha SK, Chaubey RC. Effect of gamma radiation on life history traits of Aedes aegypti (L.). Parasit Epidemiol Cont. 2016;1(2):26-35.

33. Joy TK, Arik AJ, Corby-Harris V, Johnson AA, Riehle MA. The impact of larval and adult dietary restriction on lifespan, reproduction and growth in the mosquito Aedes aegypti. Exp Gerontol. 2010;45(9):685-90.

34. Styer LM, Carey JR, Wang JL, Scott TW. Mosquitoes do sense: departure from the paradigm of constant mortality. Am J Trop Med Hyg. 2007a; 76(1):111-7.

35. Styer LM, Minnick SL, Sun AK, Scott TW. Mortality and reproductive dynamics of Aedes aegypti (Diptera: Culicidae) fed on human blood. Vect Zoon Dis. 2007b;7(1):86-98.

36. Papathanos PA, Bourtzis K, Tripet F, Bossin H, Virginio JF, Capurro ML, et al. A perspective on the need and current status of efficient sex separation methods for mosquito genetic control. Parasit Vectors. 2018; (in press)
Ready to submit your research? Choose BMC and benefit from:

- fast, convenient online submission

- thorough peer review by experienced researchers in your field

- rapid publication on acceptance

- support for research data, including large and complex data types

- gold Open Access which fosters wider collaboration and increased citations

- maximum visibility for your research: over $100 \mathrm{M}$ website views per year

At BMC, research is always in progress.

Learn more biomedcentral.com/submissions 